\title{
EFECTIVIDAD DE LAS RONDAS NOCTURNAS DE ENFERMERÍA EN LA COMODIDAD DEL PACIENTE DE UN Servicio de Cirugía
}

\section{EFFECTIVENESS OF NOCTURNAL NURSING ROUNDS IN THE COMFORT OF THE PATIENT OF A SURGERY SERVICE}

Diana Elizabeth Calle Jacinto de Guillén 1

Natalie Roxana Loncharich Vera ${ }^{2}$

1 Enfermera. Maestro en Gestión del Cuidado en Enfermería. Universidad Peruana Cayetano Heredia, Lima-Perú.

2 Enfermera. Maestro en Salud Pública. Universidad Peruana Cayetano Heredia, Lima-Perú.

Calle Jacinto de Guillén, D., \& Loncharich Vera, N. (2020). EFECTIVIDAD DE LAS RONDAS NOCTURNAS DE ENFERMERÍA EN LA COMODIDAD DEL PACIENTE DE UN SERVICIO DE CIRUGía. Revista Ene De Enfermería, 14(1). Consultado de http://www.ene-enfermeria.org/ojs/index.php/ENE/article/view/984 


\section{Resumen}

Determinar la efectividad de las rondas nocturnas de enfermería en la comodidad del paciente en un servicio de cirugía. Métodos: investigación cuantitativa y cuasi-experimental, la muestra estuvo conformada por 32 pacientes en el grupo control y 32 en el grupo experimental. Resultados: el nivel de comodidad mejoró desde un nivel medio a uno alto. Conclusión: las rondas nocturnas de enfermería mejoran la comodidad del paciente, no sólo identificando oportunamente sus necesidades y dirigiendo sus acciones a las demandas biológicas, psicoespiritual, ambiental y social del paciente, sino también organizando de manera adecuada las intervenciones de enfermería y el cuidado nocturno.

Palabras clave: Efectividad, comodidad del paciente, cuidado de enfermería, relación paciente-enfermera, cuidados nocturnos.

\section{Abstract}

Objective: to determine the effectiveness of the night rounds of nursing in the comfort of the patient in a surgery service. Methods: quantitative and quasiexperimental research, the simple consisted of 32 patients in the control group and 32 in the experimental group. Results: the comfort level improved from a medium level to a high level. Conclusion: the nocturnal nursing rounds improve the comfort of the patient, not only timely identifying their needs and directing their actions to the biological, psychospiritual, environmental and social demands of the patient, but also adequately organizing nursing interventions and night care.

Key Words: Effectiveness; patient comfort; nursing care; nurse-patient relations; night care. 
CALLE-JACINTO DE GUILLÉN - LONCHARICH-VERA

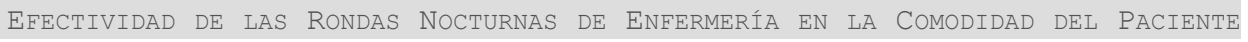

\section{INTRODUCCIÓN}

El cuidado del paciente es la esencia de la profesión de Enfermería y como actividad requiere de un valor personal y profesional que implica conocer a cada ser humano tratándolo como persona y no como patología (1). Cuando el ser humano enferma, la enfermera está obligada a prestar atención a los detalles que influyen en la comodidad de la persona durante la hospitalización, dirigiendo sus acciones no sólo a las demandas biológicas sino también a las sociales, espirituales y psíquica (1). Este fenómeno de la comodidad ha sido estudiado por Katharine Kolcaba quien plantea la valoración de las necesidades de comodidad, el diseño de medidas para satisfacer esas necesidades y su posterior valoración después de su implementación como la esencia del cuidado enfermero (2). En base a los preceptos de Kolcaba, se puede entender la comodidad como la experiencia inmediata y holística de fortalecerse gracias a la satisfacción de las necesidades de tres tipos de comodidad: alivio, tranquilidad y trascendencia, en los cuatro contextos de la experiencia humana (física, psicoespiritual, social y ambiental) (3).

La persona durante una hospitalización experimenta aquella sensación de disconformidad, la mayoría de veces por el trato recibido por parte del personal administrativo y asistencial (4). En otras ocasiones, manifiestan insatisfacción porque el personal de enfermería no mostró interés en solucionar cualquier problema durante su hospitalización; y además que el trato no fue amable, respetuoso ni con paciencia ${ }^{(5)}$.

Estas necesidades requieren ser identificadas por el profesional de enfermería a través de la valoración, la cual se pone de manifiesto con un seguimiento continuo, como con las rondas de enfermería, asegurando que los pacientes sean atendidos regularmente por una enfermera cada dos horas durante las noches, brindándoles comodidad para lograr una sensación de bienestar y mejorar o aliviar las molestias generadas por el proceso de enfermedad.

El propósito principal del estudio fue obtener una información válida y significativa sobre el nivel de comodidad que experimentan los pacientes hospitalizados con el cuidado brindado por el profesional de enfermería, para luego determinar si las rondas nocturnas de enfermería resultan efectivas priorizando la comodidad como un resultado deseable para los pacientes y demostrar, a través de la investigación, que brindar medidas de comodidad no sólo representa el cuidado integral de enfermería, sino también podría contribuir en la recuperación del paciente. 


\section{MATERIAL y MÉTOdO}

Se realizó un estudio de nivel aplicativo, con enfoque cuantitativo y cuasi-experimental. Se llevó a cabo en el servicio de cirugía mujeres del Hospital Santa Rosa, Lima-Perú.

La muestra la constituyeron 32 pacientes que ingresaron a la hospitalización en el mes de setiembre del 2017 y que conformaron el grupo control. El grupo experimental estuvo conformado también por 32 pacientes que ingresaron a la hospitalización en el mes de octubre del 2017. En ambos grupos la muestra fue seleccionada por muestreo no probabilístico, de tipo intencional o por conveniencia.

Se utilizó el cuestionario de comodidad elaborado por la investigadora principal, en base a la Teoría de Katharine Kolcaba. Este instrumento consta de dos partes, la primera contiene los datos generales y la segunda consta de 23 preguntas que se miden con una escala tipo Likert de 1 a 4. Esta escala evalúa la comodidad en cuatro contextos: físico, psicoespiritual, ambiental y social; las cuales tuvieron cuatro categorías (nunca, casi nunca, casi siempre, siempre), donde los ítems positivos tuvieron la codificación siguiente: nunca (1), pocas veces (2), varias veces (3) y siempre (4); y para los ítems negativos la codificación fue: nunca (4), pocas veces (3), varias veces (2) y siempre (1).

La validez del instrumento se realizó mediante juicio de expertos y los resultados fueron sometidos a prueba binomial dando un valor de significancia estadística inferior a $0.05(p<0.05)$. Se realizó una prueba piloto con 20 pacientes, donde el coeficiente alfa de Cronbach fue de 0.815. En términos generales, se aceptó que un valor 0.7 o mayor (alfa $>0.7$ ) será un valor satisfactorio y consecuentemente se consideró al cuestionario de comodidad como un instrumento de medición fiable.

En el mes de setiembre, al grupo control (la mayoría sometida a cirugía laparoscópica y las otras a cirugía abierta) se les aplicó la encuesta en el momento del alta médica y se determinó el nivel de comodidad durante su estancia hospitalaria. La última semana de setiembre, aquellas enfermeras que aceptaron voluntariamente participar del estudio fueron capacitadas sobre la importancia de las rondas nocturnas de enfermería para lograr la comodidad del paciente hospitalizado, y cómo deben desarrollarse durante el turno nocturno.

En el mes de octubre, durante la estancia hospitalaria del grupo experimental (la mayoría sometida a cirugía laparoscópica y las otras a cirugía abierta), se realizó las rondas nocturnas de 
CALLE-JACINTO DE GUILLÉN - LONCHARICH-VERA EFECTIVIDAD DE LAS RONDAS NOCTURNAS DE ENFERMERÍa EN LA COMOdidAd DEL PACIENTE

enfermería al inicio de cada guardia nocturna y luego cada dos horas, las cuales fueron ejecutadas por la enfermera capacitada y bajo la supervisión de la investigadora principal. Durante las rondas, la enfermera logró identificar a aquellas pacientes que no conciliaban el sueño por diversos factores: dolor, ansiedad, preocupación, entre otros; y realizaba intervenciones de enfermería que eran registradas en un formato. A aquellas pacientes que se encontraban durmiendo, no se les interrumpía su descanso. En el momento del alta médica se les aplicó el instrumento para medir el nivel de comodidad.

Ambos grupos fueron entrevistados sólo por la investigadora principal para el llenado del cuestionario de comodidad. Una vez terminada la recolección de los datos, estos fueron incorporados en una matriz creada en Microsoft Excel para su posterior análisis con el programa estadístico de libre distribución Epi Info versión 7.2.

Para determinar la efectividad de las rondas nocturnas de enfermería, se comparó el puntaje total obtenido en el cuestionario de comodidad de los pacientes del grupo control y experimental. Dadas las características de las variables se utilizó la prueba no paramétrica $\mathrm{U}$ de Mann Whitney.
$Y$ para determinar la efectividad de las rondas nocturnas de enfermería según cada contexto en ambos grupos se utilizó la prueba no paramétrica $U$ de Mann Whitney, a un nivel de significancia estadística de $0.05(p<0.05)$.

El estudio fue aprobado por el Comité Institucional de Ética de la Universidad Peruana Cayetano Heredia y por el Comité de Ética en Investigación del Hospital Santa Rosa. A las participantes se les explicó los objetivos del estudio, se solicitó el consentimiento informado por escrito, asegurando la confidencialidad, anonimato y voluntariedad de su participación, pudiendo retirarse de la investigación en cualquier momento.

\section{REsultados}

La población estuvo constituida por las pacientes hospitalizadas en un servicio de cirugía mujeres, quedando la muestra conformada por 32 mujeres del grupo control y 32 del grupo experimental. La edad media para el grupo control fue de 48,91 años y para el grupo experimental de 43.16 años. Más del $90 \%$ de pacientes fueron atendidos por la especialidad de cirugía, en su mayoría fueron sometidas a cirugía laparoscópica (colecistectomía y apendicectomía) seguida de cirugías convencionales (apendicectomía, hernioplastia, entre otros) con un 
CALLE-JACINTO DE GUILLÉN - LONCHARICH-VERA EFECTIVIDAD DE LAS RONDAS NOCTURNAS DE ENFERMERÍA EN LA COMODIDAD DEL PACIENTE

promedio de hospitalización, para ambos grupos, de 3-4 días.

Para la recolección de datos, se registraron las actividades de comodidad realizadas por el profesional de enfermería, siendo en su mayoría: aliviar el dolor, acudir a la unidad del paciente cada dos horas o a solicitud (si fuera el caso), ofrecer el urinario antes de su descanso, permitir que exprese sus sentimientos, colocar barandillas de la cama, brindar apoyo emocional, entre otros.

En términos generales, el puntaje obtenido en el grupo control es inferior al del grupo experimental, fundamentando que las rondas nocturnas de enfermería son efectivas en la comodidad del paciente en un servicio de cirugía, porque mejoró el nivel de comodidad de medio a alto en las pacientes durante su estancia hospitalaria.

Los contextos referidos al físico, psicoespiritual y ambiental mejoraron su nivel de comodidad, en función de las diversas actividades realizadas por el profesional de enfermería al identificar oportunamente las necesidades del paciente y resolverlas para facilitar el descanso nocturno de ellos.

En el contexto social se mantuvo la comodidad en un nivel medio, sugiriendo poner énfasis en aquellas necesidades del paciente que probablemente ante la hospitalización, requiera apoyo emocional y reconocer (a través de la identificación) al personal de enfermería que estará a cargo de él.

\section{Discusión}

El presente estudio demostró que las rondas nocturnas de enfermería mejoran significativamente el nivel de comodidad en los pacientes hospitalizados en un servicio de cirugía, incluso diferenciando los aspectos físico, psicoespiritual, ambiental y social. Las rondas nocturnas de enfermería no sólo identifican oportunamente las necesidades de los pacientes, sino también son efectivas sobre la calidad de atención a través de la reducción del índice de caídas y la disminución en el llamado al personal de enfermería. Esto se debe a que al satisfacer aquellas necesidades a su debido momento el paciente permanecerá tranquilo, cómodo y seguro durante el día o noche. Tal como se demuestra en el estudio "ronda horaria y caídas de los pacientes: ¿qué factores fomentan el éxito?" (6) donde luego de implementar este programa proactivo de rondas horarias orientada al paciente se redujo el índice de caídas de 3.9 a 1.3 caídas/ 1000 días de hospitalización.

$Y$ en el trabajo realizado por Saleh BS y colaboradores (7) en un centro de rehabilitación, los resultados reflejaron una reducción significativa no solo en 
el llamado al personal de enfermería, sino también se redujo el índice de caídas y un $50 \%$ de reducción en las ulceras por presión. Así mismo, la implementación del sistema de ronda de enfermería aumentó la satisfacción del paciente durante su estancia hospitalaria. Es así, que la comodidad se vuelve fundamental en la práctica de enfermería y se establece como un objetivo del plan de cuidado para satisfacer las necesidades de cada individuo, alcanzando así una asistencia más humana y singular (8).

El nivel de comodidad medio que se obtuvo en el presente estudio antes del inicio de las rondas nocturnas de enfermería difiere con el obtenido en el estudio realizado en un servicio de medicina de un hospital público en el Callao (9), donde el componente confort resultó desfavorable. Estos resultados nuevamente reflejan la importancia de identificar oportunamente las necesidades de los pacientes, no sólo en el contexto físico, sino también en el contexto ambiental, psicoespiritual y social. Siendo lo más viable, el seguimiento a través de las rondas de enfermería, que deben ser planificadas no sólo durante las noches sino también durante el día, lo cual no sólo mejorará la comodidad del paciente sino también la percepción que tiene éste respecto al cuidado de enfermería. Este seguimiento a las necesidades que presentan los pacientes durante la hospitalización, contribuye a mejorar la relación paciente-enfermera, así como la relación madre e hijo, tal como lo demuestra Álvarez-Franco en su trabajo realizado con mujeres que presentaron hemorragia postparto y describieron que las enfermeras les preguntaban frecuentemente por su comodidad, favoreciendo la relación madre-hijo y generando en ellas una sensación de seguridad (10). 
En relación al nivel de comodidad en el contexto físico y psicoespiritual, este mejoró a través de las rondas nocturnas de enfermería, porque además de priorizar la satisfacción de las necesidades físicas y/o biológicas, se organizaron los cuidados nocturnos, lo cual permitió un acercamiento entre enfermera y paciente, eliminando gradualmente aquella barrera de temor y desconfianza que se genera durante la hospitalización. Este resultado, concuerda con el estudio realizado en la unidad de cuidados intensivos de una institución de salud en Bogotá (11), refuerza la postura de que las enfermeras además de satisfacer las necesidades en el componente biológico, buscan ofrecer un cuidado psicoemocional como parte del cuidado integral que le brindan al paciente como a la familia.

Los aspectos que miden el contexto psicoespiritual reflejan un mejor nivel de comodidad en aquellas pacientes que formaron parte del grupo experimental, porque pusieron de manifiesto que durante las rondas nocturnas, el profesional de enfermería la escuchó con mayor atención, le permitió expresar sus sentimientos sobre su enfermedad y tratamiento, y sobre todo, sintió la presencia de la enfermera durante las noches, generándole un sentimiento de seguridad. Tal es así, que Quintero MT. y Gó- mez M. (12), describieron que los pacientes hospitalizados después de padecer un trauma afirmaron que no sólo requieren compensación física por parte del profesional de enfermería, sino también necesitan recibir un cuidado humanizado que contenga sentimientos de esperanza, ánimo, compañía y seguridad frente a los momentos de soledad, tristeza, dolor y angustia que estaban padeciendo. Estos resultados abren las puertas para fortalecer el cuidado individualizado que se debe brindar al paciente, mirándolo como un ser holístico que no sólo acude a un servicio de salud por una dolencia física, sino trae consigo una historia donde probablemente varias de sus necesidades sean de tipo psicológico, espiritual, emocional o social. Tal como se menciona en el estudio "Estar ahí, significado del cuidado espiritual: la mirada de los profesionales de enfermería" (13), las enfermeras más allá de cuidar, realizan múltiples acciones que tienden a buscar el bienestar espiritual de los pacientes, convirtiendo esta relación paciente-enfermera en un soporte especial ante momentos difíciles durante la hospitalización.

El nivel de comodidad en el contexto ambiental mejoró de un nivel medio a uno alto luego de las rondas nocturnas de enfermería porque el seguimiento 
CALLE-JACINTO DE GUILLÉN - LONCHARICH-VERA EFECTIVIDAD DE LAS RONDAS NOCTURNAS DE ENFERMERÍA EN LA COMODIDAD DEL PACIENTE

cada dos horas permitió realizar actividades que no sólo brindaron comodidad a través del control del ruido y evitando las interrupciones del sueño del paciente, sino que además le proporcionó seguridad física. A diferencia del estudio realizado por Ríos (14), que investigó además del uso de barandales, otras variables como uso de timbre, lámpara de cabecera, entre otros; pero igual demostró la efectividad de las intervenciones en la aplicación de medidas de seguridad con el fin de evitar eventos adversos que pongan en riesgo a los pacientes.

Otro estudio realizado por Medina y colaboradores, nos muestra que seguido del dolor y las molestias de la enfermedad, hace falta disminuir los estímulos ambientales porque son estos aspectos los que más influyen en la conciliación del sueño en el paciente hospitalizado (15). Así mismo, otros consideran importante reorganizar los cuidados durante el periodo de descanso del paciente en el turno noche, ya que el $57.2 \%$ de los pacientes hospitalizados en una unidad de medicina interna les afectaron las actuaciones del personal sanitario durante la noche (16). Esta situación podría mejorarse con una adecuada planeación del cuidado que limite al máximo las interrupciones del descanso nocturno (17).
En este estudio uno de los aspectos que midieron el contexto social, y que resulta pieza importante porque marca el inicio de la interacción entre la enfermera y el paciente, fue la presentación e identificación del profesional de enfermería ante el paciente sujeto de cuidado. Los resultados muestran que a pesar de las rondas nocturnas, el $9.4 \%$ de las pacientes manifestaron que la enfermera nunca se presentó antes de atenderlas, lo cual evidencia que aún no se consolida como un hábito esta actividad. Similar resultado muestra el estudio "Evaluación de la satisfacción con los cuidados de enfermería en el área de hospitalización de Cardiología" (18), en el cual el paciente no sabía quién era la enfermera responsable de su cuidado en cada turno, calificando este ítem como un aspecto negativo durante la hospitalización. Estos datos nos indican que no sólo es importante organizar las actividades que se realizarán durante el turno, sino resulta fundamental que el paciente reconozca quién es la enfermera que estará a cargo de su cuidado, a la que pueda acudir ante alguna duda o preocupación y que posteriormente, reflejaría una mejora en la calidad del cuidado por parte del equipo de enfermería.

Por lo anterior mencionado, se observa que la comodidad del paciente en el contexto social para ambos grupos 
CALLE-JACINTO DE GUILLÉN - LONCHARICH-VERA EFECTIVIDAD DE LAS RONDAS NOCTURNAS DE ENFERMERÍA EN LA COMODIDAD DEL PACIENTE

(control y experimental) se mantiene en un nivel medio, que, relacionado con la investigación realizada por Freitas y colaboradores (19) donde las necesidades sociales alcanzan un nivel bajo de positividad cuando son evaluados por el paciente, debido a que el equipo de enfermería le presta poca atención a estas necesidades. Esto nos lleva nuevamente a reflexionar sobre la importancia de captar aquellos aspectos sociales que pongan en riesgo innecesario la salud mental del paciente. A diferencia del estudio realizado por Gómez y Gómez (20) donde prestaron mayor atención al aspecto social, planteando como estrategia "el apoyo social" que emergió como una estrategia de afrontamiento importante que logró mitigar el impacto físico y emocional de la enfermedad en mujeres diagnosticadas de cáncer de cérvix.

Por otro lado, en nuestro estudio se encontró que el nivel de comodidad en aquellas pacientes que tuvieron una estancia hospitalaria de 3.31 días fue de nivel medio, mientras que el nivel de comodidad en las pacientes que estuvieron hospitalizadas un promedio de 4.72 días fue de nivel alto. Si bien las pacientes que obtuvieron un mejor nivel de comodidad formaron parte del grupo a quienes se les realizó las rondas nocturnas de enfermería, los resultados son similares a los encontrados en el estudio "Confort de los pacientes hospitalizados en el servicio de neurocirugía" (21), donde el paciente evidenció mayor grado de confort (bastante alto-alto) a mayores días de estancia hospitalaria.

Esto fundamenta la necesidad de considerar a la comodidad como un objetivo terapéutico que el profesional de enfermería debe hacer prevalecer durante la estancia hospitalaria del paciente.

Con estos resultados se prueba la hipótesis que las rondas nocturnas de enfermería son efectivas en la comodidad del paciente en un servicio de cirugía de un hospital general. Entonces, es preciso establecer que a través de las intervenciones de enfermería se contribuye a identificar las necesidades del paciente, mejorar la seguridad del paciente y organizar adecuadamente el cuidado de enfermería.

\section{CONCLUSIONES}

La comodidad debe ser considerada un objetivo deseable por parte del equipo de enfermería, que pasa el mayor tiempo con el paciente hospitalizado y es quien identifica diversas necesidades, prestando especial atención a aquellas que alteren el descanso nocturno y pongan en riesgo el bienestar del paciente. 
El seguimiento programado al

paciente cada dos horas, realizado a través de las rondas nocturnas de enfermería, hace visible el cuidado no sólo a través de aquellas acciones que aborden las necesidades de tipo físico, sino también aquellas de tipo psicoespiritual, ambiental y social, enfocando nuevamente el cuidado hacia lo humanizado y holístico.

Resulta importante medir la comodidad del paciente en las unidades de hospitalización para posteriormente implementar las rondas nocturnas de enfermería, ya que han demostrado mejorar el nivel de comodidad del paciente hospitalizado en un servicio de cirugía. 


\section{BIBLIOGRAFÍA}

1. Nava, M. (2010) "Estudio de caso con utilización del instrumento de Katharine Kolcaba teoría de rango medio del confort". Enfermería Neurológica, Vol. 9, № 2, México, 94-104.

2. Ferrer, E. (2009) "La medición de la comodidad en enfermeras y pacientes oncológicos". Reduca, $\mathrm{N}^{\circ}$ 2, Madrid, 501-515.

3. Guevara, M., Laverde, O. (2015) "Aplicación de la teoría de la comodidad en el baño en cama". Index de Enfermería, Vol. 24, № 1-2, Granada, de la Wold Wide Web: http://xurl.es/gznb1

4. Informe de gestión de quejas y atención al usuario. (28 de febrero de 2016). Recuperado de: https:// bit.ly/2JqootN

5. Informe de encuesta de satisfacción aplicada a los usuarios externos de los servicios de emergencia, hospitalización y consultorios externos. (08 de mayo de 2016). Recuperado de: https://bit.ly/30uxueb

6. Goldsack, J., Bergey, M., Mascioli, S., Cunningham, J. (2015) "Ronda horaria y caídas de los pacientes: ¿qué factores fomentan el éxito?". Nursing, Vol. 32, № 5, España, 61-66.

7. Saleh, B., et al. (2011) "The nursing rounds system: Effect of patient's call light use, bed sores, fall and satisfaction level". International Journal of Nursing Practice, $N^{\circ} 17$, Asia, 299-303.

8. Pott, F., et al. (2013) "Medidas de conforto e comunicação nas ações de cuidado de enfermagem ao paciente crítico". Revista Brasileira de Enfermagem, Vol. 66, $\mathrm{N}^{\circ}$ 2, Brasilia, 174-179.

9. Silva-Fhon, J. et al. (2015) "Percepción del paciente hospitalizado respecto a la atención de enfermería en un hospital público". Enfermería Universitaria, Vol. 12, N2 2, México, 80-87.

10.Alvarez, C. (2013) "Cómo describen el cuidado de enfermería las mujeres que presentaron hemorragia postparto". Aquichan, Vol. 13, № 1, Colombia, 17-26.

11.Vanegas, B. et al. (2008) "Experiencias de profesionales de Enfermería en el cuidado psicoemo- cional a pacientes en la Unidad de Cuidados Intensivos, 2006". Revista Colombiana de Enfermería, Vol. 3, N³, Colombia, 21-31.

12.Quintero, M., Gómez, M. (2010) "Nursing Care Means Help". Aquichan, Vol. 10, $\mathrm{N}^{\circ} 01$, Colombia, 8-18.

13.Uribe, S., Lagoueyte, M., (2014) "Estar ahí, significado del cuidado espiritual: la mirada de los profesionales de enfermería". Avances en Enfermería, Vol. 32, N 2, Colombia, 261-270.

14.Bentolero, L., Zamorano, M., Añorve, A. (2008) "Medidas para la seguridad física de los pacientes aplicadas en la terapia postquirúrgica". Revista mexicana de enfermería cardiológica, Vol. 16, $\mathrm{N}^{\circ}$ 1, México, 5-10.

15.Medina, A., Feria, D., Oscoz, G. (2009) "Los conocimientos sobre el sueño y los cuidados enfermeros para un buen descanso". Enfermería Global, $N^{\circ} 17$, Murcia, de la Wold Wide Web: https:// bit.ly/2HpSaww

16.Muñoz, L. et al. (2015) "Percepción de los pacientes de medicina interna de su descanso nocturno". RqR Enfermería Comunitaria, Vol. 3, № 4, Valladolid, 7-18.

17.Rodríguez, M., Arredondo, E., Pulgarín, L. (2012) "Validación de una escala de satisfacción con el cuidado nocturno de enfermería". Revista Cubana de Enfermería, Vol. 28, № 3, Cuba, 218-227.

18. Ibarrola, S. Beortegui, E., Oroviogoicoechea, C. Vasquez, M. (2011) "Evaluación de la satisfacción con los cuidados de enfermería en el área de hospitalización de cardiología". Enfermería en cardiología, Vol. 53, N² 2, España, 27-33.

19.Freitas, J. et al. (2014) "Quality of nursing care and satisfaction of patients attended at a teaching hospital". Revista Latino-Americana de Enfermagem, Vol. 22, $N^{\circ}$ 3, Brasil, 454-460.

20.Gómez, M., Lagoueyte, M. (2012) "Social support: estrategy to cope with cervical cancer". Avances en Enfermería, Vol. 30, № 1, Colombia, 32-41.

21.Uribe, A., Torrado, I., Acevedo, Y. (2012) "Comfort of patients hospitalized in the service of neurosurgery". Revista Ciencia y Cuidado, Vol. 9, № 2, España, 17-25. 


\section{TABLAS Y ANEXOS}

Tabla 1. Nivel de comodidad según contexto y puntaje obtenido

\begin{tabular}{|c|c|c|c|}
\hline Contexto & Ítems & Puntaje & Nivel de Comodidad \\
\hline Físico & 1 al 7 & $\begin{array}{c}7-13 \\
14-20 \\
21-28\end{array}$ & $\begin{array}{c}\text { Bajo } \\
\text { Medio } \\
\text { Alto }\end{array}$ \\
\hline Psicoespiritual & 8 al 12 & $\begin{array}{l}8-10 \\
11-13 \\
14-17\end{array}$ & $\begin{array}{c}\text { Bajo } \\
\text { Medio } \\
\text { Alto }\end{array}$ \\
\hline Ambiental & 13 al 19 & $\begin{array}{l}13-15 \\
16-18 \\
19-22\end{array}$ & $\begin{array}{l}\text { Bajo } \\
\text { Medio } \\
\text { Alto }\end{array}$ \\
\hline Social & 20 al 23 & $\begin{array}{c}4-7 \\
8-11 \\
12-16\end{array}$ & $\begin{array}{c}\text { Bajo } \\
\text { Medio } \\
\text { Alto }\end{array}$ \\
\hline GENERAL & 1 al 23 & $\begin{array}{l}32-48 \\
49-65 \\
66-83\end{array}$ & $\begin{array}{c}\text { Bajo } \\
\text { Medio } \\
\text { Alto }\end{array}$ \\
\hline
\end{tabular}

Fuente: datos del estudio, 2017. 
Tabla 2. Nivel de comodidad del paciente del servicio de cirugía del Hospital Santa Rosa, según el grupo control y experimental

\begin{tabular}{ccc} 
Grupo & Puntaje promedio & Nivel de comodidad \\
Control & 55,34 & Medio \\
\hline Experimental & 68,53 & Alto \\
\hline
\end{tabular}

$p<0,05$

Fuente: datos del estudio, 2017.

Tabla 3. Nivel de comodidad del paciente del servicio de cirugía del Hospital Santa Rosa, en el contexto físico según el grupo control y experimental.

\begin{tabular}{ccc} 
Grupo & Puntaje promedio & Nivel de comodidad \\
Control & 17,41 & Medio \\
\hline Experimental & 21,09 & Alto \\
\hline
\end{tabular}

$p<0,05$

Fuente: datos del estudio, 2017.

Tabla 4. Nivel de comodidad del paciente del servicio de cirugía del Hospital Santa Rosa, en el contexto psicoespiritual según el grupo control y experimental.

\begin{tabular}{ccc} 
Grupo & Puntaje promedio & Nivel de comodidad \\
\hline Control & 13,59 & Medio \\
\hline Experimental & 15,47 & Alto \\
\hline
\end{tabular}

$\mathrm{p}<0,05$

Fuente: datos del estudio, 2017. 
Tabla 5. Nivel de comodidad del paciente del servicio de cirugía del Hospital Santa Rosa, en el contexto ambiental según el grupo control y experimental.

\begin{tabular}{ccc} 
Grupo & Puntaje promedio & Nivel de comodidad \\
\hline Control & 16,22 & Medio \\
\hline Experimental & 20,44 & Alto \\
\hline
\end{tabular}

$p<0,05$

Fuente: datos del estudio, 2017.

Tabla 6. Nivel de comodidad del paciente del servicio de cirugía del Hospital Santa Rosa, en el contexto social según el grupo control y experimental.

\begin{tabular}{ccc} 
Grupo & Puntaje promedio & Nivel de comodidad \\
\hline Control & 8,13 & Medio \\
\hline Experimental & 11,53 & Medio \\
\hline
\end{tabular}

$p<0,05$

Fuente: datos del estudio, 2017. 